\title{
A Coupled Pseudospectral-Differential Quadrature Method for a Class of Hyperbolic Telegraph Equations
}

\author{
Fangzong Wang and Yong Wang \\ College of Electrical Engineering \& New Energy, China Three Gorges University, Yichang, Huibei Province 443002, China \\ Correspondence should be addressed to Yong Wang; yongwang2015wy@163.com
}

Received 16 September 2017; Revised 10 November 2017; Accepted 28 November 2017; Published 20 December 2017

Academic Editor: Francesco Marotti de Sciarra

Copyright (c) 2017 Fangzong Wang and Yong Wang. This is an open access article distributed under the Creative Commons Attribution License, which permits unrestricted use, distribution, and reproduction in any medium, provided the original work is properly cited.

\begin{abstract}
Pseudospectral methods and differential quadrature methods are two kinds of important meshless methods, both of which have been widely used in scientific and engineering calculation. The Lagrange interpolation polynomials are used as the trial function of the two methods, and the same distribution of grid points is used. This paper points out that the differential quadrature method is a special form of the pseudospectral method. On the basis of the above, a coupled pseudospectral-differential quadrature method (PSDQM) is proposed to solve a class of hyperbolic telegraph equations. Theoretical analysis and numerical tests show that the new method has spectral precision convergence in spatial domain and has A-stability in time domain. And it is suitable for solving multidimensional telegraph equations.
\end{abstract}

\section{Introduction}

The spectral methods [1] are a class of new numerical methods proposed by Gottlieb and Orszag for solving partial differential equations. As a kind of meshless methods, it can be essentially expressed as using special orthogonal polynomials (such as Chebyshev and Legendre polynomials) as test functions to discretize partial differential equations. Specifically, it is to define a set of orthogonal functions as basis functions on the calculation domain and use basis functions to approximate the desired variable as its spectral approximation form. Its notable feature is that only a few discrete points are needed to get a higher approximation accuracy [2]. Spectral methods can be divided into the Galerkin method and the spectral collocation method (SCM) or the pseudospectral method (PSM) according to the different approximation schemes [1]. For the latter, the differentiation matrix [3] is used to compute derivatives at the collocation points, which obtains great convenience for the SCM. So the differentiation matrix plays an important role in the SCM for numerical calculation [3].

The differential quadrature methods (DQM) [4] also are a class of meshless methods for solving differential or partial differential equations, which were proposed by Bellman and
Casti in the early 1970s as an extension of the idea of conventional integral quadrature. The differential quadrature method was proved to be essentially equivalent to the general collocation method [5]. The basic idea of DQM is to use the weighted linear sum of the function values of all discrete points in a coordinate direction over the whole domain to represent the derivative of the function at that discrete point. The differential quadrature method is simple in mathematical principle and its implementation does not depend on the variational principle [5]. In addition, it is also considered to be easy in programming and achieve higher computational efficiency. In order to solve the weighting coefficients or the weighting coefficients matrix of differential quadrature method, the researchers have deduced the implicit matrix expression and the explicit recursive algebraic formulation, respectively, using the general polynomial and the Lagrange interpolation polynomial as the trial function [6-8]. In [7], it was pointed out that the weighting coefficients of DQM depend only on the choice of trial function and the distribution of grid points and have nothing to do with the actual research problems.

In this study, a coupled pseudospectral-differential quadrature method is presented for solving a class of hyperbolic telegraph equations. The hyperbolic telegraph equation is first 
reduced to a set of ordinary differential equations by spatial discretization based on the spectral collocation method. Then the $s$-stage $s$-order time domain differential quadrature method is employed to solve the resulting initial value problem. Finally, the resulting algebraic equations can be solved by either direct or iterative methods. As a comparison, the method in [9] is also used for solving the telegraph equation.

The rest of the paper is arranged as follows. In Section 2, the spectral collocation method or pseudospectral method is briefly introduced. In Section 3, the space domain differential quadrature method is firstly introduced. Then, we point out the equivalence relationship of pseudospectral method and differential quadrature method in the spatial domain. In Section 4, we deduce how to use the proposed coupled pseudospectral-differential quadrature methods to solve one-dimensional and two-dimensional hyperbolic telegraph equations in detail. In Section 5, three specific numerical examples are computed, which are given to verify the computational accuracy and numerical stability of the proposed method. Conclusions are then given in Section 6.

\section{Pseudospectral Method}

Pseudospectral method, also known as the collocation points method [1, 2, 9], is an efficient and meshless numerical method for solving partial differential equation or equations. Without loss of generality, consider the differential equation $u(x)$ of a one-dimensional function:

$$
L(u(x))=f(x), \quad x \in[c, d],
$$

where $L$ is a linear differential operator and $f(x)$ is a given smooth function of space; $u(x)$ is a variable that is unknown. $x$ is one point in the interval $[c, d]$. The core idea of the spectral method and pseudospectral method is consistent; namely, the variable $u(x)$ can be interpolated and approximated by the truncated series of basis function $\phi_{n}(x)$; that is,

$$
u(x) \cong u_{N}(x)=\sum_{n=0}^{N} \alpha_{n} \phi_{n}(x)
$$

where $N$ is the number of divided intervals; $\alpha_{n}$ is the spectral coefficients. $u_{N}(x)$ is the approximate value of $u(x)$.

To measure the approximate effect of (2), define the approximate residual value $r(x, \alpha)$ as follows:

$$
r(x, \alpha)=L\left(u_{N}\right)-f(x) .
$$

We can see from the above analysis that the essential part of spectral method is to find spectral coefficients $\alpha_{n}$, which make the approximate residual values $r(x, \alpha)$ as small as possible.

In general, basis functions include discrete Fourier series, Chebyshev polynomials, Lagrange interpolation basis function, and so on [3]. The collocation points include the following four typical types (take standardized interval $[-1,1]$, for example), which are defined as follows [4]:

(1) Uniform grid points

$$
x_{i}=2 \frac{i}{N}-1, \quad i \in(0, N) \text {. }
$$

(2) Chebyshev-Gauss-Lobatto grid points

$$
x_{i}=\cos \frac{i \pi}{N}, \quad i \in(0, N)
$$

(3) Gauss-Legendre grid points

$$
x_{0}=-1 ; x_{i}, i \in(1, N-1) \text { are the zero points of } \frac{\mathrm{d}^{N-1}\left(x^{2}-1\right)^{N-1}}{\mathrm{~d} x^{N-1}}=0 ; x_{N}=1
$$

(4) Gauss-Lobatto grid points

$$
x_{i}=\frac{1}{2}\left(1-\cos \left(\frac{i \pi}{N}\right)\right), \quad i \in(0, N) \text {, }
$$

where $x_{i} \in[0,1], i \in(0, N)$. Therefore, the Gauss-Lobatto grid points on the interval $[-1,1]$ can be obtained by a linear transformation.

Using the collocation points method to obtain the approximate value of $u(x)$, each collocation point $x_{i}(i \in$ $(0, N))$ satisfies (3); that is,

$$
r\left(x_{i}, \alpha_{i}\right)=L\left(u_{N}\left(x_{i}\right)\right)-f\left(x_{i}\right)=0 .
$$

Solving the algebraic equations about spectral coefficients $\alpha_{i}$, the approximate solution $u_{N}$ of the variable $u(x)$ is obtained.
To avoid solving directly (8), consider the PSM or collocation point $s$ method with the collocation points $\left\{x_{0}, \ldots, x_{N}\right\}$ and the corresponding approximate value $\left\{u_{0}, \ldots, u_{N}\right\}$. Denote $L\left(u\left(x_{i}\right)\right)=f_{i}, i \in(0, N)$; the $p, p \geq 1$ order differential matrix $\mathbf{D}_{N}^{(p)}$ is defined in the corresponding collocation points under the linear differential operator $L$; that is,

$$
\mathbf{F}=\mathbf{D}_{N}^{(p)} \mathbf{V}
$$

where $\mathbf{F}=\left[f_{0}, \ldots, f_{N}\right]^{\mathrm{T}} ; \mathbf{V}=\left[u_{0}, \ldots, u_{N}\right]^{\mathrm{T}}$.

From formula (9), it can be seen that using the differentiation matrix $\mathbf{D}_{N}^{(p)}$ brings great convenience for numerical calculation. The solution procedure of the differentiation matrices of the pseudospectral method is given in [3] in detail. It is necessary to point out that the differentiation matrix 
formation of the pseudospectral method is related to the interpolation basis function and the collocation points, and it has nothing to do with the specific problem [3]. In other words, if basis function and collocation points are given, the unique differentiation matrices can be formed. The $p$-order differential matrix $\mathbf{D}_{N}^{(p)}$ of pseudospectral method with the Lagrange interpolation basis function and the space discrete points using the Chebyshev-Gauss-Lobatto grid points is presented as follows $[3,10]$ :

$$
\mathbf{D}_{N}^{(p)}=\left[\begin{array}{ccc}
d_{00}^{(p)} & \cdots & d_{0 N}^{(p)} \\
\vdots & \ddots & \vdots \\
d_{N 0}^{(p)} & \cdots & d_{N N}^{(p)}
\end{array}\right] \in R^{(N+1) \times(N+1)},
$$

where, in the case of $p=1$, and

$$
\begin{aligned}
d_{00}^{(1)} & =\frac{2 N^{2}+1}{6} ; \\
d_{N N}^{(1)} & =-\frac{2 N^{2}+1}{6} ; \\
d_{i i}^{(1)} & =\frac{-x_{i}}{2\left(1-x_{i}^{2}\right)}, \quad i=1,2, \ldots, N-1 ; \\
d_{i j}^{(1)} & =\frac{\gamma_{i}}{\gamma_{j}} \frac{(-1)^{i+j}}{\left(x_{i}-x_{j}\right)}, \quad i \neq j, i, j=0,1, \ldots, N-1 ; \\
\gamma_{i} & = \begin{cases}2 & i=0, N \\
1 & i=2, \ldots, N-1,\end{cases}
\end{aligned}
$$

when $N=1,2,3$, the first-order differential matrices are presented as follows:

$$
\begin{aligned}
& \mathbf{D}_{1}^{(1)}=\left[\begin{array}{rr}
\frac{1}{2} & -\frac{1}{2} \\
\frac{1}{2} & -\frac{1}{2}
\end{array}\right] ; \\
& \mathbf{D}_{2}^{(1)}=\left[\begin{array}{ccc}
\frac{3}{2} & -2 & \frac{1}{2} \\
\frac{1}{2} & 0 & -\frac{1}{2} \\
-\frac{1}{2} & 2 & -\frac{3}{2}
\end{array}\right] ; \\
& \mathbf{D}_{3}^{(1)}=\left[\begin{array}{cccc}
\frac{19}{6} & -4 & \frac{4}{3} & -\frac{1}{2} \\
1 & -\frac{1}{3} & -1 & \frac{1}{3} \\
-\frac{1}{3} & 1 & \frac{1}{3} & -1 \\
\frac{1}{2} & -\frac{4}{3} & 4 & -\frac{19}{6}
\end{array}\right] .
\end{aligned}
$$

The second-order differentiation matrix $\mathbf{D}_{N}^{(2)}$ of the PSM can be obtained directly by the following formula $[3,10]$ :

$$
\mathbf{D}_{N}^{(2)}=\mathbf{D}_{N}^{(1)} \mathbf{D}_{N}^{(1)}
$$

When $N=1,2,3$, the second-order differentiation matrices are given in the formula (13):

$$
\begin{aligned}
& \mathbf{D}_{1}^{(2)}=\left[\begin{array}{ll}
0 & 0 \\
0 & 0
\end{array}\right] ; \\
& \mathbf{D}_{2}^{(2)}=\left[\begin{array}{ccc}
1 & -2 & 1 \\
1 & -2 & 1 \\
1 & -2 & 1
\end{array}\right] ; \\
& \mathbf{D}_{2}^{(2)}=\left[\begin{array}{cccc}
\frac{16}{3} & -\frac{28}{3} & \frac{20}{3} & -\frac{8}{3} \\
\frac{10}{3} & -\frac{16}{3} & \frac{8}{3} & -\frac{2}{3} \\
-\frac{2}{3} & \frac{8}{3} & -\frac{16}{3} & \frac{10}{3} \\
-\frac{8}{3} & \frac{20}{3} & -\frac{28}{3} & \frac{16}{3}
\end{array}\right] .
\end{aligned}
$$

For convenience, the above method is named Chebyshev pseudospectral method in next sections.

\section{Differential Quadrature Method}

Differential quadrature method is also a kind of meshless numerical method [11]. Without loss of generality, suppose one-dimensional function $g(x)$ is sufficiently smooth in the whole interval $[a, b]$; and the interval $[a, b]$ is divided into $M$ sections; that is,

$$
L(g(x))=\sum_{i=0}^{M} A_{i}(x) g\left(x_{i}\right),
$$

where $L$ means linear differential operator; $A_{i}(x) i \in(0, M)$ is called the interpolation basis function; $a=x_{0} \leq x_{i} \leq x_{M}=$ $b, x_{i}$ is the $(i+1)$ th discrete point.

If $L(g(x))=g^{(p)}(x),(15)$ can be rewritten as the following formula:

$$
\left.\frac{\mathrm{d}^{p} g(x)}{\mathrm{d} x^{p}}\right|_{x=x_{i}}=\sum_{j=0}^{M} w_{i j}^{(p)} g\left(x_{j}\right), \quad i \in(0, M),
$$

where $w_{i j}^{(p)}$ is the $p$-order derivative weighting coefficient $s$ of DQM.

In DQM, using general polynomial as test functions, it is difficult to solve the weighting coefficients when $M$ increases to a certain value [8]. Using Lagrange interpolation polynomial as test functions, the explicit expression of the weighting coefficients is shown as follows (take standardized interval $[-1,1]$, for example) $[6,8]$ :

$$
\begin{aligned}
& q_{i j}=\frac{J\left(x_{i}\right)}{\left(x_{i}-x_{j}\right) J\left(x_{j}\right)}, \quad i \neq j, i, j=0,1, \ldots, M, \\
& q_{i i}=-\sum_{i=0, i \neq j}^{M} q_{i j}, \quad i=j, \quad i=0,1, \ldots, M,
\end{aligned}
$$


where $T(x)=\prod_{k=0}^{M}\left(x-x_{k}\right), J(x)=T^{\prime}(x)$.

$$
\begin{aligned}
& w_{i j}^{(p)}=p\left(q_{i j} w_{i i}^{(p-1)}-\frac{w_{i j}^{(p-1)}}{x_{i}-x_{j}}\right), \\
& i \neq j, i, j \in(0, M), \\
& w_{i i}^{(p)}=-\sum_{i=0, i \neq j}^{M} w_{i j}^{(p)}, \quad i=j, \quad i=(0, M), p \geq 2 .
\end{aligned}
$$

Shu has proved that the weighting coefficients are equivalent either using general polynomial or Lagrange interpolation polynomial as test functions.

In general, considering the grid points $\sigma_{i}, i \in(0, M)$, in the regularized interval $[-1,1]$, obviously, the transformation between $x_{i}$ and $\sigma_{i}$ can be achieved by the following linear transformation:

$$
x_{i}=\frac{b-a}{2} \sigma_{i}+\frac{b+a}{2} .
$$

Similarly, the coefficients $\xi_{i j},(i, j=0,1, \ldots, M)$ on the general interval $[a, b]$ can be obtained by using the following transformation formula [12]:

$$
\xi_{i j}=\left(\frac{2}{b-a}\right)^{p} \psi_{i j}
$$

where $\psi_{i j},(i, j=0,1, \ldots, M)$ is the $p$-order derivative weighting coefficient of the regularized region $[-1,1]$.

The weighting coefficients matrix $\mathbf{W}_{M}^{(p)}$ of the $p$-order derivative of $g(x)$ is defined as follows:

$$
\mathbf{W}_{M}^{(p)}=\left[\begin{array}{ccc}
w_{00}^{(p)} & \cdots & w_{0 M}^{(p)} \\
\vdots & \ddots & \vdots \\
w_{M 0}^{(p)} & \cdots & w_{M M}^{(p)}
\end{array}\right] \in R^{(M+1) \times(M+1)}
$$

Define $L(g(x))=g^{(p)}(x)$ and $L\left(g\left(x_{i}\right)\right)=h_{i}, i \in(0, M)$; (15) can be rewritten as the following matrix form:

$$
\mathbf{H}=\mathbf{W}_{M}^{(p)} \mathbf{G}
$$

where $\mathbf{H}=\left[h_{0}, h_{1}, \ldots, h_{M}\right]^{\mathrm{T}} ; \mathbf{G}=\left[g\left(x_{0}\right), g\left(x_{1}\right), \ldots\right.$, $\left.g\left(x_{M}\right)\right]^{\mathrm{T}}$.
When $p=1, M=1,2,3$, the first-order weighting coefficients matrices with the grid points using the Chebyshev points in the regularized region $[-1,1]$ are shown as follows:

$$
\begin{aligned}
& \mathbf{W}_{1}^{(1)}=\left[\begin{array}{rr}
\frac{1}{2} & -\frac{1}{2} \\
\frac{1}{2} & -\frac{1}{2}
\end{array}\right] ; \\
& \mathbf{W}_{2}^{(1)}=\left[\begin{array}{rrr}
\frac{3}{2} & -2 & \frac{1}{2} \\
\frac{1}{2} & 0 & -\frac{1}{2} \\
-\frac{1}{2} & 2 & -\frac{3}{2}
\end{array}\right] ; \\
& \mathbf{W}_{3}^{(1)}=\left[\begin{array}{rrrr}
\frac{19}{6} & -4 & \frac{4}{3} & -\frac{1}{2} \\
1 & -\frac{1}{3} & -1 & \frac{1}{3} \\
-\frac{1}{3} & 1 & \frac{1}{3} & -1 \\
\frac{1}{2} & -\frac{4}{3} & 4 & -\frac{19}{6}
\end{array}\right] .
\end{aligned}
$$

Similarly, the second-order derivative weighting coefficients matrices can expressed as follows:

$$
\begin{aligned}
& \mathbf{W}_{1}^{(2)}=\left[\begin{array}{ll}
0 & 0 \\
0 & 0
\end{array}\right] ; \\
& \mathbf{W}_{2}^{(2)}=\left[\begin{array}{lll}
1 & -2 & 1 \\
1 & -2 & 1 \\
1 & -2 & 1
\end{array}\right] ; \\
& \mathbf{W}_{3}^{(2)}=\left[\begin{array}{cccc}
\frac{16}{3} & -\frac{28}{3} & \frac{20}{3} & -\frac{8}{3} \\
\frac{10}{3} & -\frac{16}{3} & \frac{8}{3} & -\frac{2}{3} \\
-\frac{2}{3} & \frac{8}{3} & -\frac{16}{3} & \frac{10}{3} \\
-\frac{8}{3} & \frac{20}{3} & -\frac{28}{3} & \frac{16}{3}
\end{array}\right] .
\end{aligned}
$$

Obviously, the above DQM is mainly for the numerical computation in the spatial domain. The basic content of time domain differential quadrature method has been analyzed in detail in [5, 7]. Reference [7] indicates that classical $s$ stage $s$-order time domain DQM can be transformed into equivalent implicit Runge-Kutta method. From the definition, test functions, interpolation points, and differentiation matrix or weighting matrix of the DQM and PSM, in the spatial domain, and the differential matrices of the PSM are equivalent to the weighting coefficients matrices of the DQM as long as the test functions and the interpolation points are consistent with each other. In other words, the PSM and the DQM are also equivalent in the spatial domain.

Inspired by this, a new coupled numerical method is proposed, which is called pseudospectral-differential quadrature methods (PSDQM). In this paper, PSDQM is used to solve 
the hyperbolic telegraph equations. And the effectiveness of PSDQM is illustrated by comparing with the method used in [9].

In the following, the four-stage time domain differential quadrature method using uniform grid points will be given as an example; that is,

$$
\begin{aligned}
& \widetilde{\mathbf{W}}_{4}^{(1)}=\left[\begin{array}{cccc}
-\frac{10}{3} & 6 & -2 & \frac{1}{3} \\
-\frac{8}{3} & 0 & \frac{8}{3} & -\frac{1}{3} \\
2 & -6 & \frac{10}{3} & 1 \\
-\frac{16}{3} & 12 & -16 & \frac{25}{3}
\end{array}\right] ; \\
& \mathbf{W}_{0}=-\widetilde{\mathbf{W}}_{4}^{(1)} \mathbf{e}=\left[\begin{array}{llll}
-1 & \frac{1}{3} & -\frac{1}{3} & 1
\end{array}\right]^{\mathrm{T}},
\end{aligned}
$$

where $\mathbf{e}$ is a column vector of dimension four, all components of which are one.

\section{Solving Hyperbolic Telegraph Equations By PSDQM}

First, we can consider the following one-dimensional hyperbolic telegraph equations:

$$
\frac{\partial^{2} \chi(x, t)}{\partial t^{2}}+k_{0} \frac{\partial \chi(x, t)}{\partial t}=a_{0}^{2} \frac{\partial^{2} \chi(x, t)}{\partial x^{2}}+b_{0} \chi(x, t),
$$

where $k_{0}>0, a_{0}>0$, and $b_{0}<0$ are constants related to the transmission line parameters; variable $\chi(x, t)$ denotes voltage or current on transmission line; its domain of definition is $(x, t) \in[0, \tau] \times[0, T]$; with initial condition

$$
\begin{array}{cl}
\chi(x, 0) & =g_{1}(x), \quad x \in[0, \tau] ; \\
\chi_{t}(x, 0) & =g_{2}(x), \quad x \in[0, \tau] .
\end{array}
$$

With Dirichlet boundary condition

$$
\begin{aligned}
& \chi(0, t)=m_{1}(t), \quad t \in[0, T] ; \\
& \chi(\tau, t)=m_{2}(t), \quad t \in[0, T] .
\end{aligned}
$$

By adopting transformation $\widetilde{u}(x, t)=e^{k_{0} t / 2} \chi(x, t)$ in [13], the new one-dimensional telegraph equation is obtained by transformation; that is,

$$
\frac{\partial^{2} \widetilde{u}(x, t)}{\partial t^{2}}=a_{0}^{2} \frac{\partial^{2} \widetilde{u}(x, t)}{\partial x^{2}}+\left(b_{0}+\frac{1}{4} k_{0}^{2}\right) \tilde{u}(x, t) .
$$

With new initial condition

$$
\begin{aligned}
\widetilde{u}(x, 0) & =g_{1}(x), \quad x \in[0, \tau] ; \\
\tilde{u}_{t}(x, 0) & =g_{2}(x)+\frac{1}{2} k_{0} g_{1}(x), \quad x \in[0, \tau] .
\end{aligned}
$$

With new Dirichlet boundary condition

$$
\begin{aligned}
& \widetilde{u}(0, t)=e^{k_{0} t / 2} m_{1}(t), \quad t \in[0, T] ; \\
& \widetilde{u}(\tau, t)=e^{k_{0} t / 2} m_{2}(t), \quad t \in[0, T] .
\end{aligned}
$$

Using the PSM with Lagrange interpolation basis function and Chebyshev grid points (or uniform grid points) on the interval $[-1,1]$ used as collocation points $x_{i}$, the secondorder partial derivatives $\widetilde{u}_{x x}$ can be expressed as follows:

$$
\tilde{u}_{x x}\left(\tilde{x}_{i}, t\right)=\sum_{j=0}^{N} \widetilde{d}_{i j}^{(2)} \widetilde{u}\left(\tilde{x}_{j}, t\right), \quad i=0,1, \ldots, N,
$$

where $\widetilde{d}_{i j}^{(2)}=(2 / \tau)^{2} d_{i j}^{(2)} ; \widetilde{x}_{i}=(\tau / 2) x_{i}+\tau / 2, i \in(0, N)$.

Formula (32) is substituted into (29), and the following formula can be obtained:

$$
\frac{\mathrm{d}^{2} \widetilde{\mathbf{U}}}{\mathrm{d} t^{2}}=\left(\left(\frac{2}{\tau}\right)^{2} a_{0}^{2} \mathbf{D}_{N}^{(2)}+\left(b_{0}+\frac{1}{4} k_{0}^{2}\right) \mathbf{I}_{N+1}\right) \widetilde{\mathbf{U}}
$$

where $\widetilde{\mathbf{U}}=\left[\widetilde{u}\left(\widetilde{x}_{0}, t\right), \widetilde{u}\left(\widetilde{x}_{1}, t\right), \ldots, \widetilde{u}\left(\widetilde{x}_{N-1}, t\right), \widetilde{u}\left(\widetilde{x}_{N}, t\right)\right]^{\mathrm{T}} ; \mathbf{I}_{N+1}$ is the identity matrix of dimension $N+1$.

Let $\widetilde{\mathbf{V}}=\mathrm{d} \widetilde{\mathbf{U}} / \mathrm{d} t$; formula (33) can be reduced to the firstorder equations:

$$
\begin{aligned}
& \frac{\mathrm{d} \widetilde{\mathbf{V}}}{\mathrm{d} t}=\mathbf{S} \widetilde{\mathbf{U}}, \\
& \frac{\mathrm{d} \widetilde{\mathbf{U}}}{\mathrm{d} t}=\widetilde{\mathbf{V}}
\end{aligned}
$$

If we define $\mathbf{Y}$ as follows:

$$
\mathbf{Y}=\left[\begin{array}{c}
\widetilde{\mathbf{V}} \\
\widetilde{\mathbf{U}}
\end{array}\right]
$$

obviously, formula (34) can be rewritten as the first-order linear ordinary differential equations:

$$
\frac{\mathrm{d} \mathbf{Y}}{\mathrm{d} t}=\left[\begin{array}{cc}
\mathbf{0} & \mathbf{S} \\
\mathbf{I}_{N+1} & \mathbf{0}
\end{array}\right] \mathbf{Y}
$$

where $\mathbf{S}=(2 / \tau)^{2} a_{0}^{2} \mathbf{D}_{N}^{(2)}+\left(b_{0}+(1 / 4) k_{0}^{2}\right) \mathbf{I}_{N+1}$.

Using classic time domain DQM based on uniform grid points to solve first-order ordinary differential equation (36), the specific calculation process is given in [5] and there is no repeat here. So far, the basic idea of using PSDQM for solving one-dimensional telegraph equations is summarized as follows. First of all, by using the pseudospectral method to discretize the spatial variables and obtain a linear ordinary differential equations in time domain, then using the $s$-stage $s$-order time domain DQM based on uniform grid points to solve the obtained first-order linear equations, and, finally, using the inverse transform $\widehat{\chi}(\tilde{x}, t)=e^{-k_{0} t / 2} \tilde{u}(\tilde{x}, t)$, the numerical solution of (26) on collocation points can be obtained. 
On the basis of solving the one-dimensional telegraph equations, PSDQM is extended to solve the two-dimensional hyperbolic telegraph equations [13]; that is,

$$
\begin{aligned}
\frac{\partial^{2} \chi}{\partial t^{2}}+k_{0} \frac{\partial \chi}{\partial t} & =a_{0}^{2}\left(\frac{\partial^{2} \chi}{\partial x^{2}}+\frac{\partial^{2} \chi}{\partial y^{2}}\right)+b_{0} \chi, \\
\chi(x, y, 0) & =\widetilde{g}_{1}(x, y), \quad(x, y) \in \Omega, \\
\chi t(x, y, 0) & =\tilde{g}_{2}(x, y), \quad(x, y) \in \Omega, \\
\chi(x, y, t) & =0, \quad 0 \leq t \leq T,(x, y) \in \partial \Omega,
\end{aligned}
$$

where $\chi=\chi(x, y, t) ;(x, y) \in \Omega=[0, \tau] \times[0, \tau] ; \partial \Omega$ is a closed curve of a enclosing region named $\Omega$; the meaning of other similar variables is shown in (26).

The transformation $\widehat{u}=e^{k_{0} t / 2} \chi$ in [13] is introduced; the new two-dimensional telegraph equations are obtained as follows:

$$
\begin{aligned}
\frac{\partial^{2} \widehat{u}}{\partial t^{2}} & =a_{0}^{2}\left(\frac{\partial^{2} \widehat{u}}{\partial x^{2}}+\frac{\partial^{2} \widehat{u}}{\partial y^{2}}\right)+\left(b_{0}+\frac{1}{4} k_{0}^{2}\right) \widehat{u}, \\
\widehat{u}(x, y, 0) & =\widetilde{g}_{1}(x, y), \quad(x, y) \in \Omega, \\
\widehat{u}_{t}(x, y, 0) & =\widetilde{g}_{2}(x, y)+\frac{1}{2} k_{0} \widetilde{g}_{1}(x, y), \quad(x, y) \in \Omega, \\
\widehat{u}(x, y, t) & =0, \quad 0 \leq t \leq T, \quad(x, y) \in \partial \Omega .
\end{aligned}
$$

Using the Chebyshev pseudospectral method again, the second-order partial derivatives $\widehat{u}_{x x}$ and $\widehat{u}_{y y}$ are expressed as follows:

$$
\begin{aligned}
& \widehat{u}_{x x}\left(\widehat{x}_{i}, \widehat{y}_{k}, t\right)=\sum_{j=0}^{\widehat{N}} \widehat{d}_{i j}^{(2)} \widehat{u}\left(\widehat{x}_{j}, \widehat{y}_{k}, t\right), i=0,1, \ldots, \widehat{N}, \\
& \widehat{u}_{y y}\left(\widehat{x}_{i}, \widehat{y}_{k}, t\right)=\sum_{j=0}^{\widehat{M}} \widehat{d}_{k j}^{(2)} \widehat{u}\left(\widehat{x}_{i}, \widehat{y}_{j}, t\right), \\
& k=0,1, \ldots, \widehat{M},
\end{aligned}
$$

where $\widehat{d}_{i j}^{(2)}=(2 / \tau)^{2} d_{i j}^{(2)} ; \widehat{d}_{k j}^{(2)}=(2 / \tau)^{2} d_{k j}^{(2)} ; \widehat{x}_{i}=(\tau / 2) x_{i}+$ $\tau / 2, i \in(0, \widehat{N}) ; \widehat{y}_{k}=(\tau / 2) y_{k}+\tau / 2, k \in(0, \widehat{M})$.

On the basis of formulas (34) (36) and (39) (40), (38) can be rewritten as follows:

$$
\frac{\mathrm{d} \mathbf{z}}{\mathrm{d} t}=\left(\mathbf{I}_{\widehat{N}+1} \otimes \mathbf{B}\right) \mathbf{z}
$$

where $\mathbf{z}=\left[\mathbf{z}_{0}^{\mathrm{T}}, \mathbf{z}_{2}^{\mathrm{T}}, \ldots, \mathbf{z}_{\widehat{N}-1}^{\mathrm{T}}, \mathbf{z}_{\widehat{N}}^{\mathrm{T}}\right]^{\mathrm{T}} ; \mathbf{z}_{i}=\left[\widehat{\mathbf{V}}_{i}, \widehat{\mathbf{U}}_{i}\right]$; the symbol $\otimes$ denotes the tensor product; $\mathbf{I}_{\widehat{N}+1}$ is the identity matrix of dimension $(\widehat{N}+1) ; \widehat{\mathbf{U}}_{i}=\left[\widehat{u}\left(\widehat{x}_{i}, \widehat{y}_{1}, t\right), \widehat{u}\left(\widehat{x}_{i}, \widehat{y}_{2}, t\right), \ldots, \widehat{u}\left(\widehat{x}_{i}\right.\right.$, $\left.\left.\widehat{y}_{\widehat{M}}, t\right)\right], i \in(0, \widehat{N}) ; \widehat{\mathbf{V}}_{i}=\left\lfloor\dot{\vec{u}}\left(\widehat{x}_{i}, \widehat{y}_{1}, t\right), \dot{\vec{u}}\left(\widehat{x}_{i}, \widehat{y}_{2}, t\right), \ldots, \dot{\vec{u}}\left(\widehat{x}_{i}, \widehat{y}_{\widehat{M}}\right.\right.$, $t)\rfloor, i \in(0, \widehat{N}) ; \mathbf{B} \in R^{2(\widehat{M}+1) \times 2(\widehat{M}+1)}$, and its specific expressions can expressed as follows:

$$
\mathbf{B}=\left[\begin{array}{cc}
\mathbf{0} & \widehat{\mathbf{S}} \\
\mathbf{I}_{\widehat{M}+1} & \mathbf{0}
\end{array}\right]
$$

where $\widehat{\mathbf{S}}=(2 / \tau)^{2} a_{0}^{2} \mathbf{D}_{\widehat{M}}^{(2)}+\left(b_{0}+(1 / 4) k_{0}^{2}\right) \mathbf{I}_{\widehat{M}+1} ; \mathbf{I}_{\widehat{M}+1}$ is the identity matrix of dimension $(\widehat{M}+1)$.

Similarly, classic $s$-stage $s$-order DQM based on uniform grid points is used to solve equation (41), and the solution procedure is exactly the same as the one-dimensional telegraph equation. Finally, the inverse transformation $\bar{\chi}(\widehat{x}$, $\widehat{y}, t)=e^{-k_{0} t / 2} \widehat{u}(\widehat{x}, \widehat{y}, t)$ is employed to obtain the numerical solution of telegraph equation (37).

\section{Numerical Examples}

In this section, Lagrange interpolation polynomial is used as the test function of PSDQM. Two collocation points, Chebyshev-Gauss-Lobatto grid points and uniform grid points, are selected for PSM. In order to illustrate the effectiveness of the PSDQM, the method used in [9] is compared with the PSDQM in this paper. And the time steps of both methods are $h$. Three numerical examples are given to test the effectiveness of PSDQM and compare the numerical results of PSDQM with the methods in [9].

Example 1. Solving one-dimensional telegraph equation in formula (26), let $a_{0}=2, b_{0}=-3 \pi^{2}, k_{0}=8 \pi$, and $\tau=1$, and the initial conditions and boundary conditions are as follows:

$$
\begin{aligned}
& g_{1}(x)=\sin (\pi x) ; \\
& g_{2}(x)=-\pi \sin (\pi x) ; \\
& m_{1}(t)=m_{2}(t)=0 .
\end{aligned}
$$

The exact solution of $(26)$ is $\chi(x, t)=e^{-\pi t} \sin (\pi x)$.

In order to facilitate the analysis of the computational accuracy and convergence of the PSDQM and the method in [9], the relative errors defined in [14] are employed to evaluate the numerical results. The formula for relative error $\kappa$ for onedimensional telegraph equations is given as follows:

$$
\kappa=\left(\frac{\sum_{i=0}^{N} \sum_{j=0}^{l}\left(\chi\left(\tilde{x}_{i}, t_{j}\right)-\widehat{\chi}\left(\tilde{x}_{i}, t_{j}\right)\right)^{2}}{\sum_{i=0}^{N} \sum_{j=0}^{l} \chi^{2}\left(\tilde{x}_{i}, t_{j}\right)}\right)^{1 / 2} .
$$

Accordingly, the formula for relative error $\widetilde{\kappa}$ for twodimensional telegraph equations is as follows [14]:

$\tilde{\kappa}$

$$
=\left(\frac{\sum_{i=0}^{\widehat{N}} \sum_{k}^{\widehat{M}} \sum_{j=0}^{l}\left(\chi\left(\widehat{x}_{i}, \widehat{y}_{k}, t_{j}\right)-\bar{\chi}\left(\widehat{x}_{i}, \widehat{y}_{k}, t_{j}\right)\right)^{2}}{\sum_{i=0}^{\widehat{N}} \sum_{k=0}^{\widehat{M}} \sum_{j=0}^{l}\left(\chi\left(\widehat{x}_{i}, \widehat{y}_{k}, t_{j}\right)\right)^{2}}\right)^{1 / 2} .
$$

The selected computed parameters are $N_{1}=10, h=0.01$, and $l=T / h$. The results are shown in Table 1 , which compares the relative errors of the PSDQM $(s=4)$ in this article with the method in [9].

It can be seen that the calculation accuracy and numerical stability of PSDQM using Chebyshev-Gauss-Lobatto grid points are better than those of uniform grid points, and the relative error of the PSDQM $(s=4)$ with Chebyshev points 
TABLE 1: Relative errors of Example 1.

\begin{tabular}{lccr}
\hline$T$ & & PSDQM & Method in [9] \\
\hline 0.5 & Chebyshev points & Uniform points & $1.0160 \times 10^{-6}$ \\
1.0 & $4.2546 \times 10^{-8}$ & $3.5798 \times 10^{-8}$ & $1.2390 \times 10^{-6}$ \\
2.0 & $5.1883 \times 10^{-8}$ & $5.2462 \times 10^{-8}$ & $1.2694 \times 10^{-6}$ \\
3.0 & $5.3156 \times 10^{-8}$ & $6.2491 \times 10^{-7}$ & $1.2696 \times 10^{-6}$ \\
\hline
\end{tabular}

TABLE 2: Relative errors of Example 1 with different $N_{1}$ and $T$.

\begin{tabular}{lcc}
\hline$N_{1}$ & $T$ & Chebyshev points \\
\hline 6 & 4 & $1.6729 \times 10^{-5}$ \\
6 & 6 & $1.6729 \times 10^{-5}$ \\
6 & 8 & $1.6729 \times 10^{-5}$ \\
6 & 10 & $1.6729 \times 10^{-5}$ \\
12 & 4 & $3.4613 \times 10^{-12}$ \\
12 & 6 & $3.4613 \times 10^{-12}$ \\
12 & 8 & $3.4613 \times 10^{-12}$ \\
12 & 10 & $3.4613 \times 10^{-12}$ \\
\hline
\end{tabular}

is nearly two orders of magnitude smaller than the method in [9].

To further verify the computational accuracy of PSDQM and the numerical stability in time direction, the number of collocation points $N_{1}$ in space is varied and the time-step $h=0.001$ of DQM in the time domain remains unchanged. Figure 1 shows that the calculation accuracy of the PSDQM with Chebyshev grid points is used in spatial domain at different $N_{1}$ when $T=2$, which can be seen that the relative error is convergent in form of spectral accuracy.

Table 2 shows that, in time direction, PSDQM has unconditional stability, namely, A-stability $[7,15]$. Therefore, PSDQM is better than UDDQM [16] in time domain.

Example 2. Solving one-dimensional telegraph equation in formula (26), let $a_{0}=1 / \pi, b_{0}=-4, k_{0}=4$, and $\tau=2$, and the initial conditions and boundary conditions are as follows:

$$
\begin{aligned}
& g_{1}(x)=\cosh (\pi x) ; \\
& g_{2}(x)=-\cosh (\pi x) ; \\
& m_{1}(t)=e^{-t} ; \\
& m_{2}(t)=e^{-t} \cosh (2 \pi) .
\end{aligned}
$$

The analytic solution of $(26)$ is $\chi(x, t)=e^{-t} \cosh (\pi x)$.

Select calculation parameters: $s=4, N_{2}=18, h=0.1$, and $l=T / h$. The calculation results at this case are shown in Tables 3 and 4 . Obviously, Tables 3 and 4 further verify the conclusions given in Example 1.

Example 3. Solving the two-dimensional telegraph equation in formula (37), let $a_{0}=2, b_{0}=-3 \pi^{2}, k_{0}=8 \pi$, and $\tau=1$. The initial conditions are as follows:

$$
\begin{aligned}
& \tilde{g}_{1}(x, y)=\sin (\pi x) \sin (\pi y) ; \\
& \tilde{g}_{2}(x, y)=-\pi \sin (\pi x) \sin (\pi y) .
\end{aligned}
$$

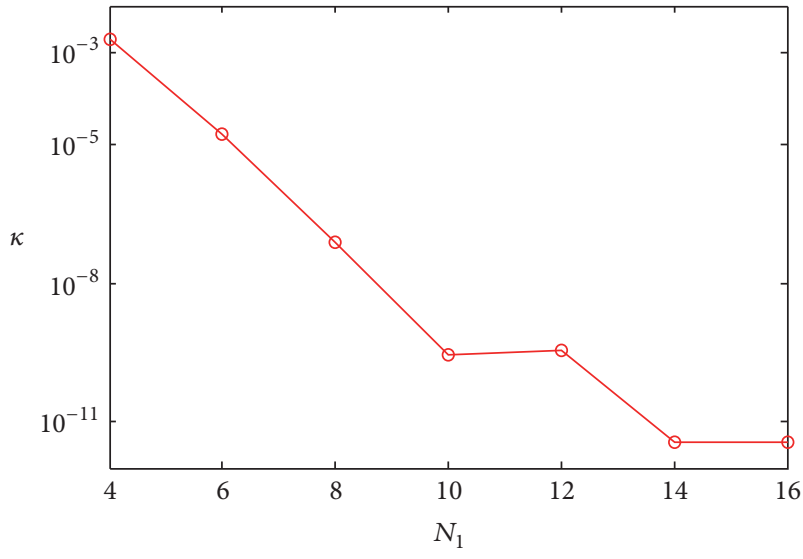

Figure 1: Relative errors of Example 1 with different $N_{1}$ at $T=2$.

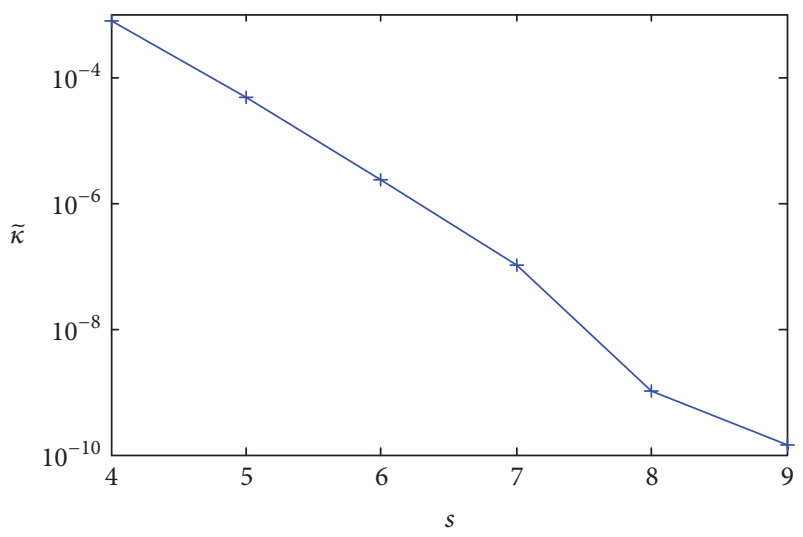

Figure 2: Relative errors of Example 3 with different $s$ at $T=3$.

In this case, the analytic solution of $(37)$ is $\chi(x, y, t)=$ $e^{-\pi t} \sin (\pi x) \sin (\pi y)$.

Select the computational parameters: $N_{3}=M_{3}=8$, $h=0.01$, and $l=T / h$. The corresponding calculation results are shown in Table 5. As can be seen from Table 5, in the two-dimensional case, the PSDQM $(s=4)$ using Chebyshev points still has higher computational accuracy than the methods in [9].

In order to compare the computational accuracy of PSDQM with different $s, N_{3}=M_{3}=12$, the time-step $h=0.1$ of DQM in time domain remains unchanged. At this situation, the results are shown in Figure 2, which shows that, with the increase of $s$ in DQM, because of the spectral accuracy in space, the accuracy of PSDQM increases with the increase of grid points' number in time domain. And this also shows the advantage of pseudospectral method. 
TABLE 3: Relative errors of Example 2.

\begin{tabular}{lccr}
\hline$T$ & & PSDQM & Method in [9] \\
\hline 0.5 & Chebyshev points & Uniform points & $1.9458 \times 10^{-7}$ \\
1.0 & $8.2086 \times 10^{-9}$ & $8.2208 \times 10^{-9}$ & $3.2409 \times 10^{-7}$ \\
2.0 & $1.3742 \times 10^{-8}$ & $1.3872 \times 10^{-8}$ & $4.6166 \times 10^{-7}$ \\
3.0 & $2.4822 \times 10^{-8}$ & $2.8261 \times 10^{-8}$ & $5.4131 \times 10^{-7}$ \\
\hline
\end{tabular}

TABLE 4: Relative errors of Example 2 with different $N_{2}$ and $T$.

\begin{tabular}{lcc}
\hline$N_{2}$ & $T$ & Chebyshev points \\
\hline 16 & 24 & $3.6662 \times 10^{-5}$ \\
16 & 26 & $3.6662 \times 10^{-5}$ \\
16 & 28 & $3.6662 \times 10^{-5}$ \\
16 & 30 & $3.6662 \times 10^{-5}$ \\
18 & 24 & $8.5851 \times 10^{-6}$ \\
18 & 26 & $8.5851 \times 10^{-6}$ \\
18 & 28 & $8.5851 \times 10^{-6}$ \\
18 & 30 & $8.5851 \times 10^{-6}$ \\
\hline
\end{tabular}

TABLE 5: Relative errors of Example 3.

\begin{tabular}{lccr}
\hline$T$ & & PSDQM & Method in [9] \\
\hline 0.5 & Chebyshev points & Uniform points & $1.0031 \times 10^{-6}$ \\
1.0 & $8.0566 \times 10^{-8}$ & $1.3426 \times 10^{-6}$ & $1.2218 \times 10^{-6}$ \\
2.0 & $8.2824 \times 10^{-8}$ & $1.5568 \times 10^{-6}$ & $1.2516 \times 10^{-6}$ \\
3.0 & $8.3131 \times 10^{-8}$ & $1.5885 \times 10^{-6}$ & $1.2518 \times 10^{-6}$ \\
\hline
\end{tabular}

TABLE 6: Relative errors of Example 3 with different $N_{3}$ and $T$.

\begin{tabular}{llc}
\hline$N_{3}$ & $T$ & Chebyshev points \\
\hline 12 & 23 & $1.3953 \times 10^{-10}$ \\
12 & 26 & $1.3953 \times 10^{-10}$ \\
12 & 29 & $1.3953 \times 10^{-10}$ \\
12 & 31 & $1.3953 \times 10^{-10}$ \\
18 & 23 & $1.4380 \times 10^{-10}$ \\
18 & 26 & $1.4380 \times 10^{-10}$ \\
18 & 29 & $1.4380 \times 10^{-10}$ \\
18 & 31 & $1.4380 \times 10^{-10}$ \\
\hline
\end{tabular}

Table 6 shows that PSDQM is unconditionally stable in the time direction and in the two-dimensional case.

\section{Conclusion}

Based on the comparison and analysis to the basic definition, interpolation points, and the test function, differentiation matrix or weighting coefficient matrix of pseudospectral method and differential quadrature method, a coupled numerical method, namely, pseudospectral-differential quadrature method (PSDQM), is proposed for solving the multidimensional telegraph equations. And this paper pointed out the equivalence of the two meshfree methods in the space domain. By analyzing three specific numerical examples, the numerical results show that PSDQM has spectral accuracy convergence in space domain and high accuracy and A-stability in time domain. In general, the coupled method inherits the advantages of the pseudospectral method and differential quadrature method, and the two methods can be mutually beneficial. In all, the PSDQM is suitable for solving multidimensional telegraph equations.

\section{Conflicts of Interest}

The authors declare that there are no conflicts of interest regarding the publication of this paper.

\section{Acknowledgments}

The authors gratefully acknowledge the support from the National Natural Science Foundation of China (NSFC) through its Grant 51377098.

\section{References}

[1] D. Gottlieb and S. A. Orszag, Numerical Analysis of Spectral Methods: Theory and Applications, Society for Industrial and Applied Mathematics, Philadelphia, PA, USA, 1977.

[2] S. A. Orszag, "Comparison of pseudospectral and spectral approximation," Studies in Applied Mathematics, vol. 51, no. 3, pp. 253-259, 1972. 
[3] B. Fornberg, A Practical Guide to Pseudospectral Method, Cambridge University Press, London, UK, 1998.

[4] R. Bellman and J. Casti, "Differential quadrature and long-term integration," Journal of Mathematical Analysis and Applications, vol. 34, pp. 235-238, 1971.

[5] J. Liu and X. W. Wang, "Comparisons of successive integration method based on differential quadrature with commonly used time integration schemes," Chinese Quarterly of Mechanics, vol. 29, no. 2, pp. 304-309, 2008.

[6] C. Shu, Differential Quadrature and Its Application in Engineering, Springer, Berlin, Germany, 2000.

[7] F. Z. Wang, X. B. Liao, and X. Xie, "Characteristics of the differential quadrature method and its improvement," Chinese Journal of Computational Mechanics, vol. 32, no. 6, pp. 765-771, 2015.

[8] C. Shu and Y. T. Chew, "On the equivalence of generalized differential quadrature and highest order finite difference scheme," Computer Methods Applied Mechanics and Engineering, vol. 155, no. 3-4, pp. 249-260, 1998.

[9] M. Javidi and A. Golbabai, "Spectral collocation method for parabolic partial differential equations with Neumann boundary conditions," Applied Mathematical Sciences, vol. 1, no. 5-8, pp. 211-218, 2007.

[10] L. N. Trefethen, Spectral Methods in Matlab, SIAM, Philadelphia, PA, USA, 2000.

[11] R. Ding and X. R. Wei, "Local differential quadrature method based on the Uzawa algorithm for the elastic-plastic torsion problems," Chinese Journal of Computational Mechanics, vol. 28, no. 4, pp. 499-503, 2011.

[12] J. Izadian, F. Nateghi, and M. Jalili, "Comparison of spectral and differential quadrature methods for solving the Burger-Huxley equation," Communications in Numerical Analysis, vol. 2013, pp. 1-7, 2013.

[13] Y. Lu and Y. Jiang, "Symplectic schemes for telegraph equations," Journal of Computational Mathematics, vol. 34, no. 3, pp. 285299, 2016.

[14] T. Liu and W. Ma, "Barycentric lagrange interpolation collocation method for two-dimensional hyperbolic telegraph equation," Chinese Journal of Computational Physics, vol. 33, no. 3, pp. 341-348, 2016.

[15] F. Z. Wang, Numerical Method for Transient Stability Computation of Large-Scale Power System, Science Press, Beijing, China, 2013.

[16] A. Jaber Ali Al-Saif, "An efficient scheme of differential quadrature based on upwind difference for solving two-dimensional heat transfer problems," Applied and Computational Mathematics, vol. 4, no. 4, pp. 275-285, 2015. 


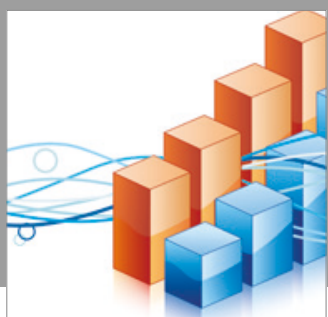

Advances in

Operations Research

vatersals

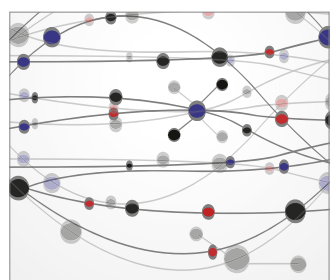

\section{The Scientific} World Journal
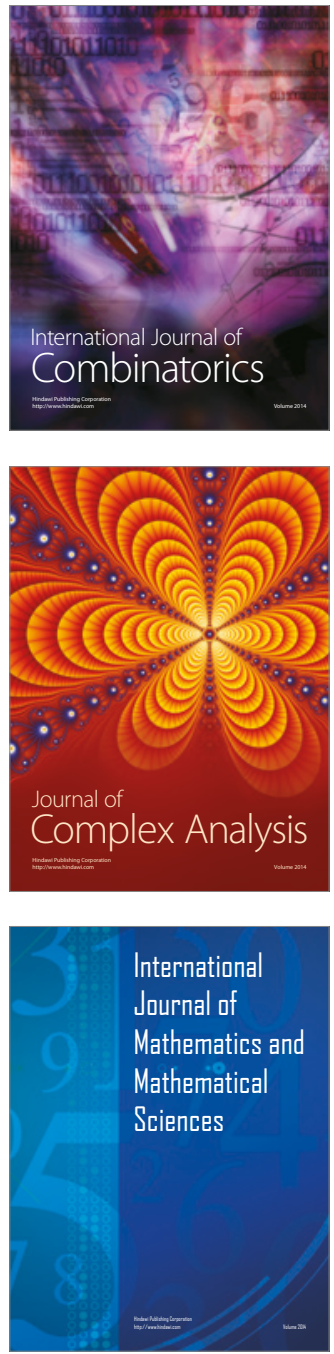
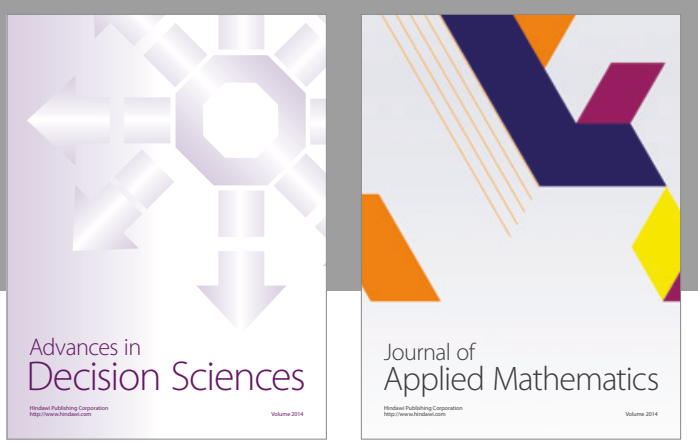

Algebra

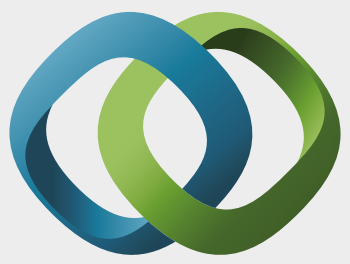

\section{Hindawi}

Submit your manuscripts at

https://www.hindawi.com
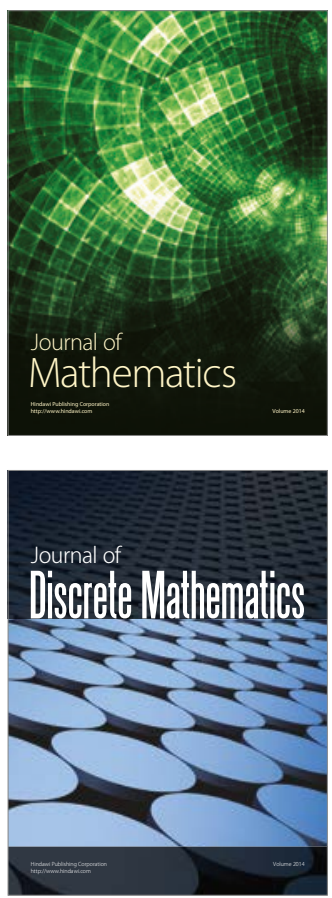

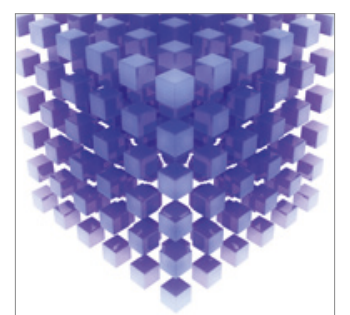

Mathematical Problems in Engineering
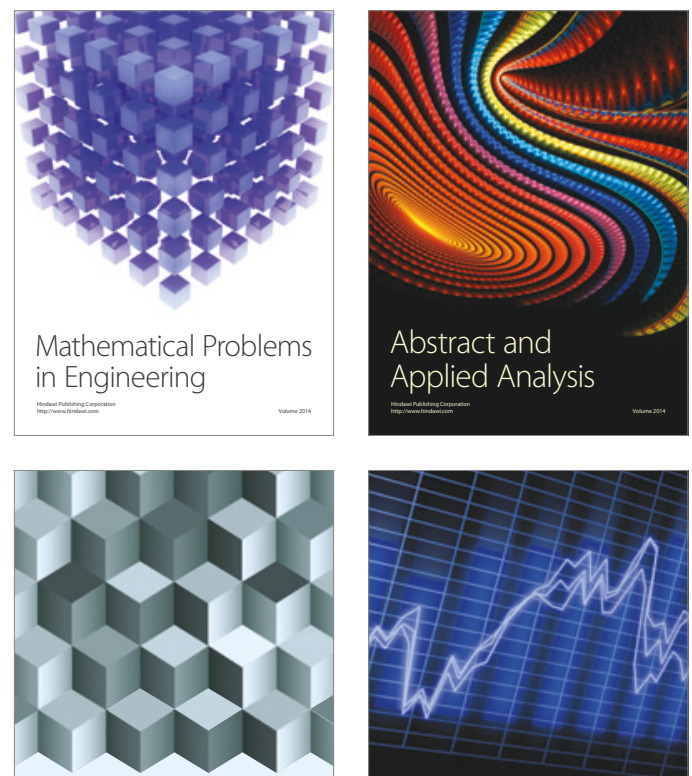

Journal of

Function Spaces

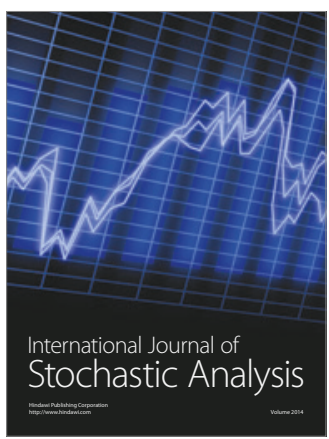

Probability and Statistics
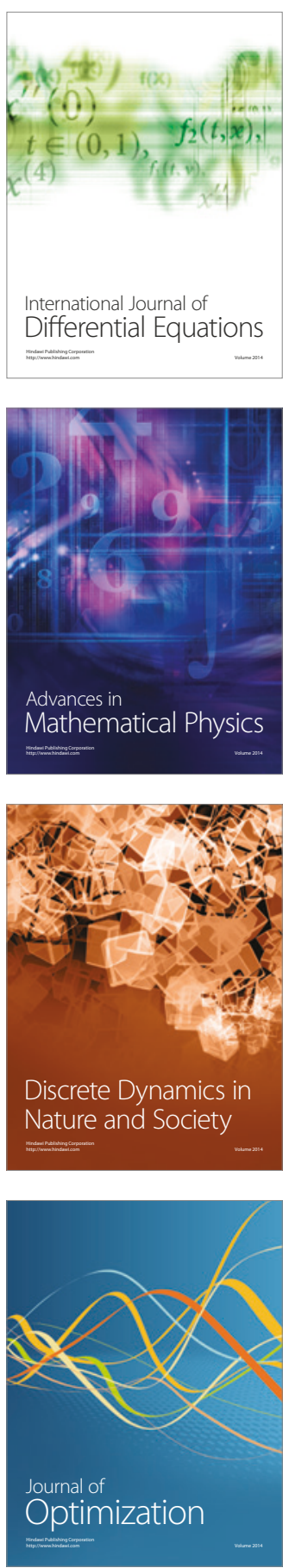